\title{
Graft Versus Host Diseases Presenting Endoscopically as a Tubular Structure Mimicking a Worm
}

\author{
Amer A Alkhatib $^{\mathrm{a}, \mathrm{b}}$, Maia M Ahdab
}

\section{To the Editor:}

Graft Versus Host Diseases (GVHD) is the leading cause of morbidity and mortality after allogenic hematopoietic stem cell transplantation, occurring in up to $75 \%$ of patients [1]. According to the degree of involvement in each of the organ systems, acute GVHD can be clinically classified as grades I - IV [2]. Colonoscopic examination can show diffuse edema, hyperemia, patchy erosion, scattered ulcer, sloughing and active bleeding [3, 4]. We report a case of a patient with GVHD that presented endoscopically as a tubular structure that looked like a dead worm.

A 63 years old female patient with history of acute myelogenous leukemia underwent unrelated donor graft rejection followed by matched unrelated donor peripheral blood stem cell transplant presented with diarrhea. The patient underwent colonoscopy that showed mildly congested and erythematous colonic mucosa. Biopsy from the colon showed focal crypt abscess, apoptotic bodies, cryptal destruction and laminar propria fibrosis consistent with graft versus host disease grade 2. CT of the abdomen and pelvis was done and showed a bowel wall thickening due to edema involving multiple loops of jejunum, however the mucosa remained enhancing. The patient was diagnosed based on the clinical presentation with severe gut GVHD.

Because the patient continued to have diarrhea and work up for infectious diarrhea was negative, a repeated colonoscopy was done and showed a granular mucosa with ulcerations involving the entire the colon. A tubular struc-

Manuscript accepted for publication August 11, 2009

${ }^{a}$ Division of Gastroenterology, Department of Medicine, University of Utah, Salt Lake City, Utah, USA

${ }^{\mathrm{b}}$ Corresponding Author: 30 North 1900 East, SOM4R118, Department of Internal Medicine, Division of Gastroenterology, University of Utah, Salt Lake City, Utah, USA. khatibamer@yahoo.com

doi:10.4021/gr2009.10.1320
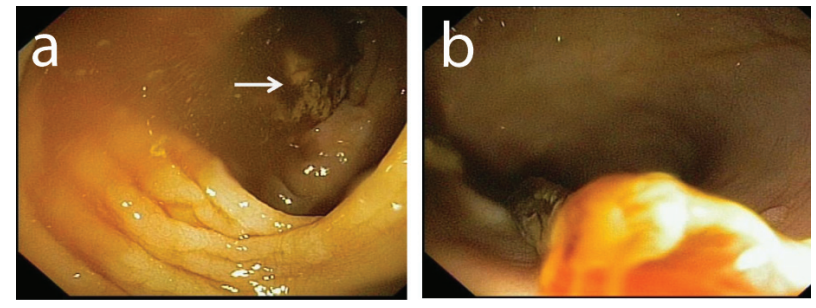

Figure 1. (a) Endoscopic image showing a floating tubular structure inside the colon; (b) Endoscopic image showing the tubular structure is attached to the colonoscopy.
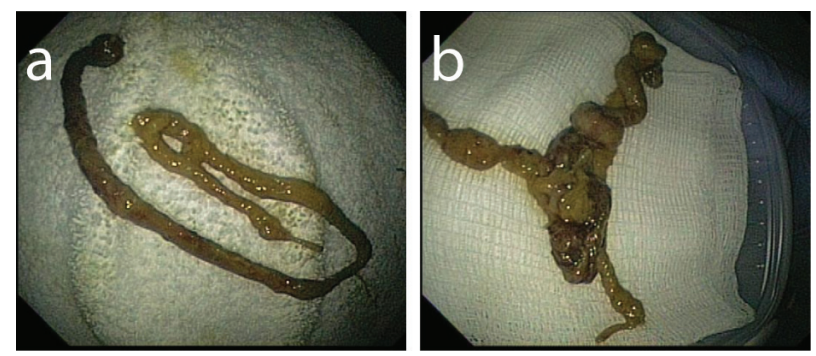

Figure 2. $(a, b)$ Images of the tubular structure that mimic worm on a towel.

ture was seen floating in the colon and looked like a worm (Fig. 1). It was $30 \mathrm{~cm}$ in length with $0.7 \mathrm{~cm}$ in diameter. It was retrieved by the colonoscopy (Fig. 2). A cross sectioning revealed fibrous tissue bands surrounded by clotted and hemorrhagic tissue. The colonic biopsy showed GVHD. The histopathological examination of the tubular structure was consistent with fibrinopurlent exudate.

We concluded that the tubular structure represented a sloughed gut tissue induced by severe gut GVHD.

\section{References}

1. Ferrara JL, Deeg HJ. Graft-versus-host disease. N Engl J Med 1991;324(10):667-674.

2. Izumi N, Furukawa T, Sato N, Okazuka K, Tsukada N, Abe T, Yano T, et al. Risk factors for acute graft-versus- 
host disease after allogeneic hematopoietic stem cell transplantation: retrospective analysis of 73 patients who received cyclosporin A. Bone Marrow Transplant 2007;40(9):875-880.

3. Xu CF, Zhu LX, Xu XM, Chen WC, Wu DP. Endoscopic diagnosis of gastrointestinal graft-versus-host disease.
World J Gastroenterol 2008;14(14):2262-2267.

4. Harada K, Higaki S, Hashimoto K, Hashimoto S, Oga A, Gondo T, Sakaida I. Study on the colonoscopic features of GVHD enteritis that developed after hematopoietic stem cell transplantation. Hepatogastroenterology 2007;54(80):2221-2227 